\title{
Advances in Nutraceuticals
}

\author{
Iyen, Ifeoma Sandra*, Ogofotha, Godwin Oghenekeno, Oko, John Odiba \\ Chemical Sciences Department, Federal University Wukari, Nigeria, P. M.B, 1020, Wukari, Nigeria \\ ${ }^{*}$ Corresponding Author: Ifeoma Sandra, Chemical Sciences Department, Federal University Wukari, \\ Nigeria, P. M.B, 1020, Wukari, Nigeria
}

\begin{abstract}
Nutraceuticals are products, other than nutrition which are also used as medicine. A nutraceutical product may be defined as a substance, which has physiological benefit or provides protection against chronic disease. Nutraceuticals may be used to improve health, delay the aging process, prevent chronic diseases, increase life expectancy, or support the structure or function of the body. Nowadays, nutraceuticals have received considerable interest due to potential nutritional, safety and therapeutic effects. Recent studies have shown promising results for these compounds in various compilations. In the present review much effort has been devoted to present new concepts about nutraceuticals based on their diseases modifying indications. Emphasis has been made to present herbal nutraceuticals effective on hard curative disorders related to oxidative stress including allergy, Alzheimer, cardiovascular, cancer, diabetes, eye, immune, inflammatory and Parkinson's diseases as well as obesity
\end{abstract}

\section{INTRODUCTION}

Nutraceutic is a term derived from "nutrition" and "pharmaceutics." The term is applied to products that are isolated from herbal products, dietary supplements (nutrients), specific diets, and processed foods such as cereals, soups, and beverages that other than nutrition are also used as medicine (Kalra, 2003).

In the US, the term "nutraceutical" products are regulated as drugs, food ingredients and dietary supplements. The term is not defined the same in different countries, but is usually defined as a product isolated from foods that is generally sold in medicinal forms not usually associated with food. A nutraceutical product may be defined as a substance, which has physiological benefit or provides protection against chronic diseases. Nutraceuticals may be used to improve health, delay the aging process, prevent chronic diseases, increase life expectancy, or support the structure or function of the body (Zhaoj, 2007).

Nutraceuticals, in contrast to pharmaceuticals, are substances, which usually have not patent protection. Both pharmaceutical and nutraceutical compounds might be used to cure or prevent diseases, but only pharmaceutical compounds have governmental sanction (Chauhan et al., 2013). A dietary supplement is considered as a product that bears or contains one or more of the following dietary ingredients: A mineral, a vitamin, an amino acid, a medical herb or other botanical, a dietary substance for use by man to supplement the diet by increasing the total daily intake, or a concentrate, metabolite, constituent, extract, or combinations of these ingredients. Nutraceuticals are of these nutritional supplements which are used for health purposes other than nutrition (Zeisel, 1999). Some popular nutraceuticals include ginseng, Echinacea, green tea, glucosamine, omega-3, lutein, folic acid, and cod liver oil. Majority of the nutraceuticals possess multiple therapeutic properties.

Nowadays, nutraceuticals have received considerable interest due to potential nutritional, safety and therapeutic effects. A market research recently proposed that the worldwide nutraceuticals market is expanding and would reach US $\$ 250$ billion by 2019 (Hardy, 2000).

Recent studies have shown promising results for these compounds in various pathological complications such as diabetes, atherosclerosis, cardiovascular diseases (CVDs), cancer, and neurological disorders. These conditions involve many changes, including alterations redox state. Most of nutraceuticals have antioxidant activity with the ability to counteract this situation. Hence, they are considered as healthy sources of health promotion, especially for prevention of life threatening diseases such as diabetes, infection, renal, and gastrointestinal disorders (KhosraviBoroujeni, et al., 2012) 
To present recent advances based on their diseases modifying indications. Emphasis has been made to present herbal nutraceuticals effective on hard curative disorders related to oxidative stress, including allergy, Alzheimer, cardiovascular, cancer, diabetes, eye, immune, inflammatory and Parkinson's diseases, as well as obesity (Shirzad et al., 2011).

\subsection{History and Rediscovery of Nutrients as Nutraceuticals}

"Food may possess the ability to prevent diseases or treatment of ailment" this belief is couple of centuries old-proclaimed by our ancestors. The ancient writings and artworks of Egyptians, Romans, and Greek civilizations depicted the medicinal and spiritual applications of plants. The idea arose 3000 years ago when Hippocrates developed a relationship between food for health and therapeutic applications of it. The principal truth depicted in his statement that "Let food be thy medicine and medicine be thy food" is widely applied nowadays (Ahmad et al., 2013). Thus, from such finding, it could be believed that our nature and surroundings have much of the natural therapies to offer. One such finding presents the botanicals, which are in use from ancient times for the treatment of cancer. Similarly, there are many plant derived chemotherapies which consist of Vinca and Taxus brevifolia species to treat cancer and related problems. Furthermore, Bogdanov (2015) revealed that ginseng has been another such traditional drug which is in use from past 2000 years in China. It was from the time of Liang dynasty of China that the chemotherapy features of ginseng were discovered and applied even in today's time. From the documented history, Egyptians identified the medicinal importance of coriander, fennel, juniper, cumin, garlic, turmeric, thyme, curry, and dried mint found in pyramids. The value of such medicinal sources was so high that even cinnamon was considered more precious in comparison to gold in Egypt. Due to the explored properties of plants, the Roman emperor Heliogabalus made use of cinnamon, clove, and pepper in meals which are used in cuisines and soft drinks until present time. In addition, there were many other plants and food additives identified such as honey and certain vitamins. Honey has always been given prime importance since ancient period. It was referred in Sumerians tablet writing as one of the remedies for health problems. According to Bible, the wise Solomon has said, "Eat honey, my son, because it is good" (Old Testament, proverb 24:13). With the illustration of properties possessed by honey, apitherapy has been developed as a separate medicinal branch related with medication properties provided by honey .It has promised to offer its action as a vasodilator, anti-allergic and antihypertensive agent. In addition, other than honey, the requirement of vitamin, iodine, etc., was also noticed by the ancient people. Another instance which explains the importance of the food and nutrients within is of the ancient crewmen. During the past few centuries, many crewmen on long voyages died of scurvy, the exploring facts revealed the absence of Vitamins B and C in the diet acting as antiscorbutic which was later ensured to be the part of the diet. In the same way, Goiter was found as the common problem due to deficiency of iodine in salt (Sarin et al., 2010). The health problems, thus, were identified and were dealt with the help of diet and healthy lifestyle; therefore, all these examples very clearly explain the requirement of a healthy diet and herbal components with the potential to serve the same. One of the surveys in United Kingdom, Germany, and France has concluded that the diet has more profound effects for healthy body and mind than exercise or genetic factors (Pandey et al., 2010). The above-mentioned findings motivate and direct the research toward a rediscovery of nutrients as nutraceuticals. The researchers in past 20 years have concluded that the diet plays a key role in the prophylaxis of chronic disorders atherosclerosis and cancer. The credible success in isolation of food components had been achieved precisely with the help of proper clinical and laboratory investigations to verify the efficacy (Shirwailar et al., 2011)

\subsection{Categories of Nutraceuticals}

Nutraceuticals are non-specific biological therapies used to promote wellness, prevent malignant processes and control symptoms. These can be grouped into the following three broad categories

1) Substances with established nutritional functions, such as vitamins, minerals, amino acids and fatty acids - Nutrients

2) Herbs or botanical products as concentrates and extracts - herbals

3) Reagents derived from other sources (e.g. pyruvate, chondroitin sulphate, steroid hormone precursors) serving specific functions, such as sports nutrition, weight-loss supplements and meal replacements - Dietary supplements (Hathcock 2001) 


\subsubsection{Nutrients}

The most commonly known nutrients are antioxidant, water and fat-soluble vitamins. Many potential benefits have been attributed to antioxidant use in the form of dietary intake or supplementation (Mobarlan 1994). High dietary intake of vitamin E may prevent Parkinson's disease, determined that the oxidized form of vitamin C, dehydroascorbic acid, readily crosses the blood brain barrier (Agus et al., 1997). These findings have implications for increasing the uptake of antioxidants in the central nervous system; thus, some feel that this has the potential for improving the treatment of Alzheimer's disease found that the combination of vitamin E, C and beta carotene has been useful in reducing low density lipoprotein oxidation and subsequent atherosclerosis (Jialal and Fuller 1995)

Vitamin supplement is associated with increased antibody titre response to both hepatitis B and tetanus vaccines as a result of macrophage and $T$ cell stimulation (Klien et al., 2000). Those genetically predisposed to pancreatic cancer have low serum levels of selenium; thus, it is assumed that supplementation with selenium may help to prevent this condition (Mathew et al., 1996). Those suffering from asthma and skin cancer have also been evaluated with selenium for its potential use, although results have been inconclusive. L-arginine is a semi-essential amino acid that is a substrate for nitric oxide production demonstrated that supplementation of L-arginine improved exercise capacity in patients, who had angina (Ceremuzynski et al., 1997).

\subsubsection{Herbals}

Herbals are as old as human civilization and they have provided a complete storehouse of remedies to cure acute and chronic diseases. The knowledge of herbals has accumulated over thousands of years so that today we possess many effective means of ensuring health care. Numerous nutraceuticals are present in medicinal herbs as key components. Herbal extracts, including b-sitosterols (found in Saw Palmetto berry), cernilton (pollen extract), and pygeum africum (African plum) have been clinically evaluated for use in the treatment of benign prostatic hyperplasia (Breakman 1994). It has been found that common herbal treatment is in the use of Echinacea for the prevention and treatment of colds and flu. A series of five placebo controlled studies evaluating the use of Echinacea produced mixed results, which the authors attribute to either the use of healthy volunteers, rather than patients, or the use of extracts that were not standardized or chemically defined monopreparations (Melchart et al., 1994). Ernst suggested that St John's wort is efficacious for mild to moderate depression, but serious concern exists about its interactions with several conventional drugs (Ernst 2002).

\subsection{Classification of Nutraceuticals}

The aim of achievement of maximal state of well-being is alarmingly increased with the introduction of nutraceuticals (Dureja 2003). The term nutraceuticals, itself is a wide class which include many categories and subcategories under it. The contribution of nutraceuticals to the public health is one of the most concerned approaches in health-care system. Although the system claims to have advanced techniques to treat the diseases, still the nutraceutical approach counts because of the power it holds to treat diseases through diet. For a better explanation of comparison, pharmaceuticals include drugs for the treatment of diseases, but nutraceuticals are proposed to prevent diseases (Rajasekaran et al., 2008). Pharmaceuticals enjoy patent protection and government sanctioning whereas nutraceuticals never receive government appreciation and cannot be patented. "Referring nutraceuticals as functional foods" this idea is under discussion because of consideration of food and medicine as the same aspect. Functional food has provision to include vitamins, fats, proteins, carbohydrates for the survival of health. However, when that food is aiding in prevention and cure of ailment other than fulfilling deficiency conditions then these are called as nutraceuticals (Yadav et al., 2012). The illustration is that black cohosh is found native to a region of North America and has been employed as a medicament to treat problems related to menopause, premenstrual syndrome, and menstrual cramping. This herbal formulation comprises extracts from roots and is adopted as female tonic in case of menstrual discomfort. Thus, it holds a medicinal property to respond to a health related problem and thus can act as a nutraceutical. There is another category called as Medical food, which are therapeutic agents meant for nutritional management of a disease. An example includes preparations for managing inborn amino acid metabolism errors. Besides, there are some dietary supplements also which enhances the diet containing vitamins, minerals or concentrate, and metabolite or extract (Singh et al., 2012). All these categorized food products, plants and sources are thus very imperative to be recognized as these possess nutritional and medicinal properties. In recent years, the diet and lifestyle related disorders have become a major issue for the health care. The increased attention and awareness 
among the subjects for health care along with the availability of sophisticated methodology for determination of the nutritional level of diet is worth studying (Guptas et al., 2010). It can be reviewed from various studies that nutraceuticals provide non-specific biological therapy for treatment and prophylaxis of various diseases. To understand the applications, the nutraceuticals are needed to be classified. According to Dev et al (2011) the classification into various classes depending on their uses are:

- Traditional nutraceuticals

- Nontraditional nutraceuticals

- Fortified nutraceuticals

- Recombinant nutraceuticals

- Potential and established nutraceuticals

- Phytochemicals

- Herbals

- Functional foods

- Dietary supplements and dietary fibers

- Probiotics and prebiotics.

1.3.1. Traditional Nutraceuticals

The category consists of the food which does not undergo any manual changes. The components are natural and are having some potential which are actively involved in health benefits. Lycopene, a constituent of tomatoes is an example of this category (Chintale et al., 2013).

\subsubsection{Nontraditional Nutraceuticals}

Boosting of nutritional content by addition of nutrients, dietary components for improvement of quality of nutrition comprise this category of nutraceuticals. Beta carotene enriched rice is an example of this class (Sapkale et al., 2012).

\subsubsection{Fortified Nutraceuticals}

Fortification of food components is the process of addition of micronutrients (essential trace elements and vitamins) to food for enhancing the effectiveness and nutritional value. Its example includes milk fortified with cholecalciferol used in Vitamin D deficiency (Sinha 2012).

\subsubsection{Recombinant Nutraceuticals}

It involves the application of biotechnology and genetic engineering in the production of energy providing foods such as yoghurt and cheese or extraction of bioactive components by enzymatic or fermentation technology. Gold kiwi fruit is genetically modified for a high level of ascorbic acid, Carotenoids, and Lutein and Zeaxanthin (Beck et al., 2011).

\subsubsection{Potential and Established Nutraceuticals}

Potential nutraceuticals hold an assurance of medicinal benefits. These nutraceuticals have become established medicines only after sufficient data demonstration and clinical testing for their efficacy and safety. All nutraceuticals are potential nutraceuticals but all potential nutraceuticals are not established ones (Pandey et al 2010)

\subsubsection{Phytochemicals}

These are the chemical constituents of plants with distinct biological action. These are been reported to have active components which exerts their effects toward the metabolism and biochemical reactions in living beings and thus, provide health benefits. The herbs possessing medicinal values to be implicated in treatment and prevention of ailments are been included in the class. Botanical products may consist of fresh plant used or any part such as dried leaf, fruit, stem, seeds, roots, or concentrated extract (Dillard 2000).

\subsubsection{Functional Foods}

Functional foods are the source of absolutely necessary nutrients providing more than the quantities required for maintenance, growth, and development. The term is specially retained for food or food components that carry the evidence to provide an advantageous factors for health beyond basic nutrition (Spano 2010). The class of functional food includes many further subclasses such as cereals, 
legumes, and fermented food. The potentiality of the functional food including cereals such as rice, corn, wheat, millets, sorghum, and buckwheat has been found in many ways to eliminate the risk of coronary heart disease, tumor incidence, and lowering of blood pressure. Similar to cereals, legumes are the other subclass of functional foods which form a chief element in traditional and modern dietary patterns (Bahadoran 2014). These are highly nutritious and rich in biologically valuable proteins, bioactive peptides, and amino acids. The examples are kidney beans, split beans, chickpeas, lentils, and soybeans. These have been explored to have profound antioxidant and protective effect against cardiovascular diseases and diabetes (Preuss 2009). In addition, chocolate has also been found to be a subclass of functional food which is a richest source of proteins, calcium, iron, magnesium, and riboflavin. Citrus fruits are another type of functional food which have already been reported to produce therapeutic effects as anticancer, antiviral, antioxidant agents, and further have potential to stimulate immune system (Vishal 2012). The fermented milk and related products are also the example of a functional food which offers good digestive effects. For instance, Yoghurt is a highly nutritive fermented food associated with the anticancer activity; it has also been reported to prevent gastrointestinal infections and atherosclerosis. It is recommended for lactose intolerant patients (Shiby 2013). Further classification includes honey, which is a natural sweetener and believed to possess nutritional and medicinal value. It is composed of monosaccharide fructose, glucose, enzyme diastase, amino acids, vitamins, minerals, and aroma compounds. Its biological actions are reported in case of Type 2 diabetes, obesity and also to provide infant nutrition (Doner 1997). It also has shown the positive results for improvement of renal functions, as antioxidant and as antimicrobial agent (Elliott et al.,2002). Colostrum is another functional food which is referred to first milk secreted in parturition. It contains lactoprotein and lactalbumin due to which it is different from milk secreted later. It is rich in antibodies which provide passive immunity to a newborn in addition to proteins, immunoglobulins, and growth factors. Recent demonstrations reported its role in the treatment of autoimmune disorders. Extending further, meat products can also be included under the class of functional food. Proteins derived from soyabeans have been employed in comminuted meat products as meat replacements ( Fernandez-Gines 2013).

\subsubsection{Dietary Supplements and Dietary Fibers}

A dietary supplement is a product which comprises a supplementary dietary ingredient added as a remedy to deficiencies or diseases. The inclination toward dietary supplements has raised many folds to improve health, fitness, tonic to delay aging, improve performance, and body building. A dietary ingredient is one which enhances the food and its nutritional assessment. Vitamins and minerals as dietary supplements exist in multiple ingredients or single ingredient products in the market (Pandey 2011). Demand of dietary supplements is alarmingly increasing in developing countries such as Brazil, China, India, and Russia and it has already reserved its place in developed parts of many countries (Norman et al., 2010). Dietary supplements other than vitamins and minerals also involve herb, botanicals, amino acids, pure extracts, concentrate or combination of number of ingredients gland extracts, and organ tissues. It is not supposed to be used as traditional or conventional food element or as the regular item in the meals or diet (Pandy et al., 2011). Dietary fibers and high fiber products are of great interest because of significant health benefits. According to American Association of Cereal Chemists (AACC) (2013), it is defined as edible part of plant or carbohydrate analogous which is resistant to digestion and absorption in the small intestine. These products normalize the intestinal transit time. Its sources include brown rice, banana, cereals, oats, dry beans, and legumes (Cheung 2013).

\subsubsection{Probiotics and Prebiotics}

Probiotic category includes the live microbial food ingredients which are advantageous to health. Their action includes adhesion to gastrointestinal tract at specific sites and their survival lead to elimination of pathogens (Quigley 2000). Prebiotic category includes selectively fermented ingredients or a fiber that promote changes in gastrointestinal microflora and its activity providing good effects to the health of host. They are the fertilizing agents for the probiotic bacteria in colon. These are not affected by gastric $\mathrm{pH}$ and digestive acids. The example includes inulin which on further hydrolysis gives oligofructose and galactooligosaccharide (Shukla 2014).

\subsection{Toxicity Potential of Nutraceuticals}

A large number of people believe that nutraceuticals, especially medicinal plants, are important remedies to address health issues with no side-effects. This belief has been raised from the fact that 
they have been used for a long period without serious toxicities. Although this is true for a wide variety of nutraceuticals and they generally have less side effects in comparison to pharmaceuticals, but conventional medicine is considered that if a drug is to be effective, inevitably, it will have toxic or side-effects. The medical establishment considers herbal medicines as drugs, and as such, they must have side effects. Therefore, they need to be prepared with correct ingredients and use with caution, too (Baradaran 2012).

People consume thousands of species of plants and other nutraceuticals to meet their basic nutritional needs, but only a limited number of them have received significant safety studies. Many remain poorly understood and largely undeveloped, and their wild relatives are threatened with extinction and in need of conservation attention. Stewardship of these valuable plant resources will require rigorous science combined with an approach that respects and values traditional knowledge systems (Nasri et al., 2014).

\subsection{Antitoxicity of Nutraceuticals}

Most of the synthetic drugs possess toxicity properties, and nutraceutical compounds, particularly herbal nutraceuticals have been investigated for their potential in combating the toxic effects of toxins and other medications (Heidarian 2013). Although the toxicology of drugs is complex, there is great evidence for involvement of oxidative stress in the toxicity of a wide variety of drugs. Most of plants possess antioxidant activity and other than various specific ways to combat toxins and synthetic drugs, they generally may reduce their toxicity by reduction of oxidative stress. Kidney and liver are two organs which more than others are involved in toxic effects of other drugs as well as toxins. In this regard there are a wide variety of studies investigating the protective activities of nutraceuticals, especially medicinal plants against toxins and other drugs and promising results have been achieved (Nasrietal.,2014).

\subsection{Advances in Nutraceuticals}

\subsubsection{Cancer and Nutraceuticals}

Cancer has emerged as a major public health problem in developing countries. According to the World Cancer Report (2018) the cancer rates are increasing and it would be 15 million new cases in the year 2020 that is, a rise in 50\%. But with the consumption of Nutraceuticals the estimated new cases is predicted to reduce by $14.5 \%$. A healthy lifestyle and diet can help in prevention of cancer. Carotenoids are a group of phytochemicals responsible for different colors of the foods. They have antioxidant activities and effective on cancer prevention. Recent interest in carotenoids has focused on the role of lycopene in human health, especially in cancer disease (Willis 2003). Plants rich in daidzein, biochanin, isoflavones and genistein, also inhibit prostate cancer cell growth. Because of the unsaturated nature of lycopene it is considered to be a potent antioxidant and a singlet oxygen quencher. Lycopene concentrates in the prostate, testes, skin and adrenal where it protects against cancer. The linkage between carotenoids and prevention of cancer and $\mathrm{CAD}$, heightened the importance of vegetable and fruits in human diet (Shirzad et al., 2013). Lycopene contained vegetables and fruits exert cancer-protective effect via a decrease in oxidative stress and damage to DNA. Lycopene is one of the major carotenoids and is found exclusively in tomatoes, guava, pink grapefruit, water melon and papaya (Stahl 2005). $\beta$-carotene has antioxidant activity and prevents cancer and other diseases. Among the carotenes, $\beta$-carotene has the most antioxidant activity. Alphacarotene possesses $50-54 \%$ of the antioxidant activity of $\beta$-carotene, whereas epsilon carotene has $42-50 \%$ of the antioxidant activity (Sies 2005).

Chronic inflammation is associated with a high cancer risk. Chronic inflammation is also associated with immune-suppression, which is a risk factor for cancer. Ginseng is an example of an antiinflammatory molecule that targets many of the key players in the inflammation-to-cancer sequence (Shirad 2011).

Nowadays, phytochemicals with cancer-preventive properties have been on high attention. Chemopreventive components in fruits and vegetables, among other beneficial health effect, have potential anticarcinogenic and antimutagenic activities. A broad range of phyto-pharmaceuticals with a claimed hormonal activity, called "phyto-estrogens," is recommended for prevention of prostate and breast cancers (Limer 2004).

Citrus fruit flavonoids are able to protect against cancer by acting as antioxidants. Soyfoods are a unique dietary source of isoflavones, the polyphenolic phytochemicals exemplified by 
epigallocatechin gallate from tea, curcumin from curry and soya isoflavones possess cancer chemopreventive properties. Soybean seems to offer protection against breast, uterine, lung, colorectal, and prostate cancers. $\beta$-carotene found in yellow, orange, and green leafy vegetables and fruits such as tomatoes, lettuce, oranges, sweet potatoes, broccoli, cantaloupe, carrots, spinach, and winter squash has anticancer activity (Thomasset 2007)

Saponins are reported to possess antimutagenic and antitumor activities and might lower the risk of human cancers, by preventing cancer cells from growing. Saponins are phytochemicals which can be found in peas, soybeans, and some herbs with names indicating foaming properties such as soapberry, soapwort and soapbark. They are also present in tomatoes, potatoes, alfalfa, spinach, and clover. Commercial saponins are extracted mainly from Yucca schidigera and Quillaja saponaria (Li et al., 2003).

Tannins also scavenge harmful free radicals and detoxify carcinogens. Tannins present in grapes, lentils, tea, blackberries, blueberries and cranberries is a proven anticarcinogen is used in alternative medicine and to prevent cancer. Ellagic acid, present in walnuts, pecans, strawberries, cranberries, pomegranates and red raspberry seeds, is an anticancer agent (Liu 2003).

Pectin is a soluble fiber found in apples has been shown to prevent prostate cancer metastasis by inhibiting the cancer cells from adhering to other cells in the body. Several studies have shown that pectin decreases serum cholesterol levels. Naturally occurring phenolic acid derivatives are reported to possess potential anticancer properties. Phenolic compounds such as curcumin, gallic acids, ferulic and caffeic acid are reported to possess anticancer activity (Nasri 2014).

Glucosinolates and their hydrolysis products, including indoles and isothiocyanates, and high intake of cruciferous vegetables has been associated with lower risk of colorectal and lung cancer. Biotransformation products of glucosinolates include dithiol thiones, isothiocyanates, and sulforaphane. They block the enzymes that promote tumor growth, particularly in liver, colon, lung, breast, stomach and esophagus (Higdon 2007).

The sulfur compounds, in garlic have been found to boost the immune system and reduce atherogenesis and platelet stickiness and cancer. Sulforaphane rich in broccoli is a potent phase 2 enzyme inducer. It produces D-glucarolactone, a significant inhibitor of breast cancer. Sulforaphane is an antioxidant and stimulator of natural detoxifying enzymes. Sulforaphane has been reported to reduce the risk of breast cancer and prostate cancer (Tamadon 2014).

Curcumin is a polyphenol derived from the plant Curcuma longa, commonly called turmeric. Curcumin has been reported to possess antioxidative, anticarcinogenic, and antiinflammatory properties (Tavafi 2014).

Consumption of fruits and vegetables having cysteine, glutathione, selenium, Vitamin E, Vitamin C, lycopene, and various phytochemicals elevates the levels of antioxidative capacity. However, more investigations are needed to determine their beneficial effects in cancer prevention or treatment (Rafieian-Kopaei 2013).

Large scale clinical trials suggest that some agents such as green tea, Vitamins D and E, selenium, lycopene, soy, anti-inflammatory and inhibitors of 5a-reductase are effective in preventing prostate cancer. Cancer was not prevented by $\beta$-carotene, $\mathrm{N}$-acetylcysteine, $\alpha$-tocopherol, retinol, retinyl palmitate, or isotretinoin in smokers. Ongoing trials may help define new avenues for chemoprevention.

Several studies have shown the values of alternative and complementary medicine as adjuvant to chemotherapy or radiotherapy. Complimentary therapy may be reliable and useful supportive measure for prostate cancer patients. Majority of the studies have shown a preventive role for nutraceuticals in cancer, however more elaborate studies are needed (Brouns 2002).

\subsubsection{Diabetes and Nutraceuticals}

The most common form of diabetes is type 2 diabetes with $95 \%$ prevalence and is associated with obesity. Although various drugs for prevention and treatment of diabetes have been introduced, however, globally the total number of people with diabetes with various causes is increasing. Diabetes, not only imposes considerable economic burdens on individual patients and their families but also places substantial economic burdens on society (Baradaran 2012). In recent years a wide range of herbal dietary supplements and herbal medicines have scientifically proven to benefit type 2 
diabetes mellitus in preclinical studies, however, few have been proven to do so in properly designed randomized clinical trials (Tolouian 2013).

Isoflavones, are phytoestrogens which have structural/functional similarities to human estrogen. Soy isoflavones have been studied most and their consumption have been associated with lower incidence and mortality rate of type II diabetes, heart disease, osteoporosis and certain cancers (Fuller 1991).

Omega-3 fatty acids have been suggested to reduce glucose tolerance in patients predisposed to diabetes. For the synthesis of a long chain $n-3$ fatty acids, insulin is required; the heart may thus be particularly susceptible to their depletion in diabetes. Ethyl esters of $n-3$ fatty acids may be potential beneficial in diabetic patients (Sirtori 2002).

Lipoic acid is an antioxidant which is used for the treatment of diabetic neuropathy and seems to be effective as a long-term dietary supplement for protection of diabetics from complications. Dietary fibers from psyllium have been used extensively both as pharmacological supplements, food ingredients, in processed food to aid weight reduction, for glucose control in diabetic patients and to reduce lipid levels in hyperlipidemia. A lot of plants extracts such as Toucrium polium, cinnamon and bitter melon have been shown to prevent or treat diabetes (Momeni 2012).

\subsubsection{Eye Disorders and Nutraceuticals}

Healthy lifestyle with a diet containing foods rich in antioxidants, such as Poly unsaturated fatty acid (n-3 fatty acids), lutein and zeaxanthin appears beneficial for age-related macular degeneration (AMD). High content of polyphenolic flavonoids in nutraceuticals have been shown to possess antioxidant activity. Herbs or herbal extracts, such as green tea, Allium spp., Vitamins C and E, polyphenols, carotenoids (mainly lycopene and $\beta$-carotene), and coenzyme Q10 possess antioxidant properties and effective in AMD (Brouns 2014).

Astaxanthin is an important naturally occurring carotenoid in the marine world such as sea bream, salmon, trout, and shrimps. It possesses a number of essential biological functions such as protecting against oxidation process, protecting against ultra violet light effects, immune response and pigmentation, in aquatic animals. It is also a very potent antioxidant. Astaxanthin offers powerful protection for the eyes and prevents macular degeneration. Astaxanthin protects heart from oxidative damage, protects the nervous system from degenerative diseases like $\mathrm{AD}$ and boosts immune system function (Brookmeyers et al.,2007).

Lutein is a carotenoid which is found in many vegetables and fruits including sweet potatoes, carrots, squash, tomatoes, mangoes, corn, and leafy greens such as kale andcollards. Lutein and Zeaxanthin are used for the treatment of visual disorders. Food sources of zeaxanthin, include egg yolks, corn, green vegetables and fruits, such as brussel sprouts, cabbage, kale, broccoli, green beans, green peas, lettuce, kiwi, collard greens, spinach, and honeydew lutein and zeaxanthin also occur in plants in the form of mono- and diesters of fatty acids. A new source of these carotenoids is marigold flower (Tagetes erecta) that contains approximately $86 \%$ by weight of the carotenoids zeaxanthin and lutein (Johnson 2007).

\subsubsection{Immune System and Nutraceuticals}

A wide variety of nutraceuticals have been shown to impose crucial roles in immune status and susceptibility to some diseases conditions. Nutraceuticals that belong to the category of immune boosters are useful to improve immune function. They include extracts from the coneflowers, or herbs of the genus Echinacea, such as Echinacea angustfolia, Echinacea pillida, Echinacea purpurea. The coneflowers in particular are a popular herbal remedy used in the central United States, an area to which they are indigenous. Astragalus mongolicus, Astragalus membranaceus, and other herbs of the Astragalus genus are also effective immune boosters. Astragalus stimulates development and transformation of stem cells in the marrow and lymph tissue to active immune cells. Phytoestrogens mostly are recommended for prevention of various diseases related to hormonal imbalance. There is a special interest in soy isoflavones as potential superior alternatives to the synthetic selective estrogen receptor modulators, which are currently applied in hormone replacement therapy. Garlic and morphine also are good example of the nutraceuticals, which respectively stimulate and suppress immune system (Limer 2004). The effect of herbal medicines and bacteria on the immune system and intestinal epithelial cell function has led to new credence for the use of nutraceuticals and probiotics in clinical settings. Probiotics are effective in conditions such as infectious diarrhea in children and recurrent Clostridium difficile induced infections (Gupta et al., 2000). 
Supplementation with probiotics (live viable microbial organisms) may provide maturational signals for the lymphoid tissue and improve the balance of pro and anti-inflammatory cytokines. Probiotics manipulate the intestinal microflora to maintain a normal balance between pathogenic and nonpathogenic bacteria. Usage of these agents in the treatment of specific diseases has evolved into the ability to very high index of safety, reduction of antibiotic use and the public's positive perception about "alternative" or "natural" therapies. Most probiotic preparations are comprised of one or more lactic acid bacteria. Within this group, strains of Lactobacillus, Bifidobacterium sp. and occasionally Streptococcus are most commonly used (Fuller 1991).

\subsubsection{Cardiovascular Diseases and Nutraceuticals}

Worldwide, the prevalence of Cardiovascular Diseases (CVD) and the researches in this area is increasing. CVD is a term which is used for disorders of the heart and blood vessels and includes coronary heart disease (heart attack), peripheral vascular diseases, cerebrovascular disease (stroke), hypertension, heart failure, and so on. It is believed that low intake of vegetables and fruits is associated with a high mortality in CVD. Majority of the CVD are preventable. Many studies have reported a protective role for a diet rich in vegetables and fruits against CVD ( $\mathrm{Hu} 2002$ ). Nutraceuticals in the form of vitamins, minerals, antioxidants, dietary fibers and omega-3 polyunsaturated fatty acids ( $n-3$ PUFAs) together with physical exercise are recommended for prevention and treatment of CVD. The molecules such as polyphenols alter cellular metabolism and signaling, which is believed to reduce arterial disease (Shahbazian 2013).

Flavonoids are widely distributed in vegetables, onion, endives, cruciferous, grapefruits, apples, cherries, pomegranate, berries, black grapes, and red wine, and are available as flavones, flavanones and flavonols, playing a major role in prevention and curing the CVD (Gharipour et al., 2013). Flavonoids block the angiotensin-converting enzyme, block the cyclooxygenase enzymes that break down prostaglandins, and prevent platelet aggregation. They also protect the vascular system that carries oxygen and nutrients to cells. Anthocyanins, tannins (proanthocyanidins), tetrahydro- $\beta$ carbolines, stilbenes, dietary indoleamines, serotonin and melatonin, in plant foods are hypothesized to impose health benefits (Iriti 2006). Orange juice containing pulp is rich in flavonoids. Hesperidin is a flavanone glycoside which is classified as a citrus bioflavonoid. Citrus sinensis and tangelos are the richest dietary sources of hesperidin. The peel and membranous parts of lemons and oranges have the highest hesperidin concentrations. Hesperidin is used for the treatment of venous insufficiency and hemorrhoids (Garg et al.,2001).

Flavonoid intake was significantly inversely associated with mortality from coronary heart disease and the incidence of myocardial infarction. Flavonoids in regularly consumed foods may reduce the risk of death from coronary heart disease, especially in elderly people (Rafieian-Kopaci et al., 2013).

The rhizome of zingiber officinalis is a common condiment for various foods and beverages. It has a long history of medicinal use and has a positive effect on CVD. Ginger has potent antioxidant and antiinflammatory activities and recently it has been recommended for various diseases including hypertension and palpitation. This plant has a good protective effect on toxicity of synthetic drugs, too.

Phytosterols compete with dietary cholesterol by blocking the uptake as well as facilitating its excretion from the body. Hence, they have the potential to reduce the morbidity and mortality of CVD. Phytosterols occur in most plant species and although green and yellow vegetables contain significant amounts of sterols, their seeds concentrate them (Gita 2004).

Buckwheat seeds possess phytosterols, flavonoids, flavones, proteins and thiamin-binding proteins, etc., Buckwheat proteins lower blood cholesterol and hypertension. Dietary fibers have also cholesterol-lowering property with beneficial effects in prevention and alleviation of CVD and diabetes. Fatty acids of the omega-3 series (n-3 fatty acids) present in fish are dietary components affecting plasma lipids and the CVD, like arrhythmias. Octacosanol, present in whole grains, fruits and leaves of many plants, has lipid lowering property, with no side-effects (Sidhu 2003).

\subsubsection{Allergy and Nutraceuticals}

Allergy is a hypersensitivity disorder of the immune system. An allergic reaction usually occurs when a person's immune system reacts to normally harmless substances. Allergic reactions are distinctive because of excessive activation of certain white blood cells called mast cells and basophils by a type 
of antibody called immunoglobulin E. This reaction results in an inflammatory response which can range from uncomfortable to dangerous (Grammatikos 2008). Quercetin protects low-density lipoprotein (LDL-C) from becoming damaged, especially to blood vessels. LDL-C is an underlying cause of heart disease and quercetin acts as an antioxidant and scavenges free radicals. Diabetic patients are at higher risk of blood vessel damage from oxidative stress. Therefore, quercetin is beneficial in these patients too (Kruger et al., 2002).

\subsubsection{Alzheimer's Disease and Nutraceuticals}

Alzheimer's disease (AD) is the most common form of dementia. There is no cure for the disease and eventually leads to death. Most often, AD is diagnosed in people over 65 years of age, although the less-prevalent early-onset Alzheimer's can occur much earlier. There were 26.6 million sufferers worldwide in 2006 and is predicted to affect 1 in 85 people globally by 2050 (Brookmeyer et al., 2007).

Women are more affected in comparison to men, at a ratio of almost 2:1. Several lines of evidence suggest that oxidative stress might be related to a number of neurodegenerative disorders including AD. Nutraceutical antioxidants such as curcumin, lutein, lycopene, turmeric and $\beta$-carotene may exert positive effects on specific diseases by combating oxidative stress. The growing trends in nutraceutical usage are due to the belief that these compounds are able to postpone the development of dementias such as AD. There are several recently published papers showing the positive effects of different nutraceutical plants such as Zizyphus jujube, Lavandula officinalis on AD, learning or memory (Rabiei et al., 2014).

\subsubsection{Parkinson's Disease and Nutraceuticals}

Parkinson's disease is a degenerative disorder of the central nervous system, which its motor symptoms result from the destruction of dopamine-generating cells in the substantia nigra, with unknown causes. The most obvious symptoms are movement-related including rigidity, slowness of movement, shaking and difficulty with walking and gait. The symptoms in advanced stages of the disease include thinking and behavioral problems. Depression is the most common psychiatric symptom and symptoms include sensory, emotional and sleep problems. Parkinson's disease is more common in older people, with most cases occurring after the age of 50 (Losso 2003).

Although at present there are not sufficient scientific data to recommend nutritional supplements for Parkinson's disease, some of these supplements have shown some promising results in preliminary studies. Vitamin E, glutathione, and creatine seem to be protective against Parkinson's disease (Anwar et al., 2007).

\subsubsection{Obesity and Nutraceuticals}

Obesity is, nowadays, a global public health problem with about 315 million people involved. Obesity is a risk factor for many disorders such as hypertension, congestive heart failure, angina pectoris, hyperlipidemia, respiratory disorders, osteoarthritis, cancer, renal vein thrombosis and reduced fertility (Caterson 2002).

One of the primary causes of obesity is the increased availability of high-fat, energy-dense foods. There is a very high prevalence of obesity globally and hence nutrition and exercise play a key role in its prevention and treatment. Nutraceutical interventions are currently being investigated on a largescale basis as potential treatments for obesity and weight management. Nutraceuticals such as capsaicin conjugated linoleic acid, Momordica charantia and Psyllium fiber possess potential antiobese properties (Rubin 1994).

Although excessive consumption of energy-rich foods such as snacks, processed foods and drinks causes weight gain, however, caloric restriction and increased physical activity has been shown to be only moderately successful in managing obesity. Therefore, researchers and obese individuals are seeking the help of nutraceuticals and pharmaceuticals to prevent or treat obesity. An effective nutraceutical that can increase energy expenditure and/or decrease caloric intake is desirable for body weight reduction. Herbal stimulants, such as caffeine, ephedrine, chitosan, ma huang-guarana, and green tea are effective in facilitating body weight loss. However, their use is controversial due to their ability to cause side-effects. Green tea extract and 5-hydroxytryptophan may promote weight loss, while the former increases the energy expenditure, the latter decreases appetite (Boozer et al., 2001). 


\subsubsection{Inflammation and Nutraceuticals}

Inflammation is characterized by swelling, pain, redness and heat, and is the response of body tissues to irritation or injury. Nutraceuticals that their influence on osteoarthritis has been tested are ginger, soybean, unsaponifiable, glucosamine, chondroitin, S-adenosylmethionine. Although they are safe and well tolerated, however, the results are hampered by heterogeneity of the studies and inconsistent results. Vitamins $\mathrm{C}$ and D are micronutrients for which evidence of benefit exists. Cat's claw is a potent antiinflammatory agent. Scientists have attributed the efficacy of cat's claw to compounds called oxindole alkaloids; however, water-soluble cat's claw extracts that do not contain significant amounts of alkaloids do not possess strong antioxidant and antiinflammatory effects (Rafieian-Kopaei 2014).

Resveratrol that is present in the fruits of Vaccinium myrtillus, Vaccinium angustifolium, Vaccinium ashei, and Vaccinium corymbosum shows the strongest sirtuin-like deacetylase action of any known phytochemical. Sirtuins are chemicals that inhibit cyclooxygenase-1 enzyme and can extend the lifespan of yeast and fruit flies. They possess antiinflammatory and antifungal activities (Jang et al., 1997).

The omega- 3 and omega- 6 series have a significant role on diseases by generating potent modulatory molecules for inflammatory responses, including prostaglandins, leukotrienes, and interleukins. Gamma linolenic acid (GLA) is produced in the body from linoleic acid, an essential fatty acid of omega-6 series. GLA is a nutraceutical used for treating problems with inflammation and autoimmune diseases. Preformed GLA is present in trace amounts in nuts, green leafy vegetables, vegetable oils, such as seed oil, borage oil, Oenothera biennis oil, blackcurrant and hemp seed oil. GLA is metabolized to dihomogamma linlenic acid which undergoes oxidative metabolism by lipoxygenase and cyclooxygenase enzymes to produce antiinflammatory eicosanoids (Rouhi-Broujeni et al., 2013).

Herbal nutraceuticals with antiinflammatory activity are also available. Gentianine, present in Gentian root, is an effective antiinflammatory agent. Bromolain, a proteolytic enzyme found in extracts of stinging nettle, turmeric, pineapple, teas and extracts of turmeric or curcumin has antiinflammatory activity (Nasri et al., 2014).

Osteoarthritis is a debilitating joint disorder which affects the number of population. In 2004, the costs associated with all forms of arthritis were approximately 86 billion dollars. Joint discomfort from any joint disorders may reduce physical activity in subjects, resulting in energy imbalance and weight gain. Increased weight can exacerbate existing problems, through additional stress on joints (RouhiBroujeni 2013).

Glucosamine and chondroitin sulfate are widely used to alleviate symptoms of osteoarthritis. These nutraceuticals seem to regulate gene expression and synthesis of NO and PGE2, providing a plausible explanation for their antiinflammatory activities (Gharipour et al., 2013).

\subsection{Structures of some Common Natural Products Compounds (Nutraceuticals)}

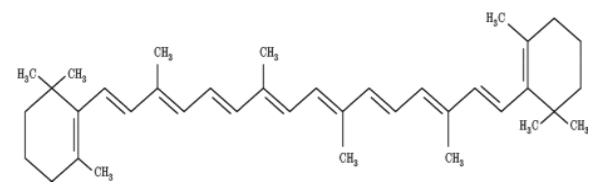

Fig1. $\beta$-Carotene

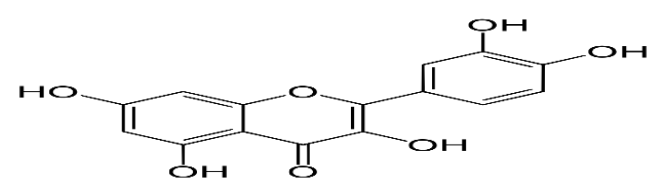

Fig2. Quercetin

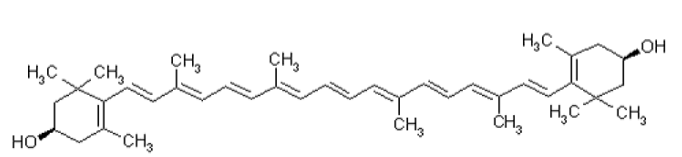

Fig3. Zeaxanthin<smiles></smiles>

Fig5. Daidzein

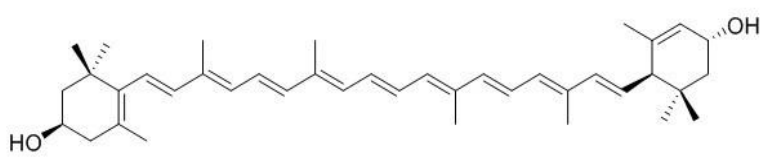

Fig4. Lutein

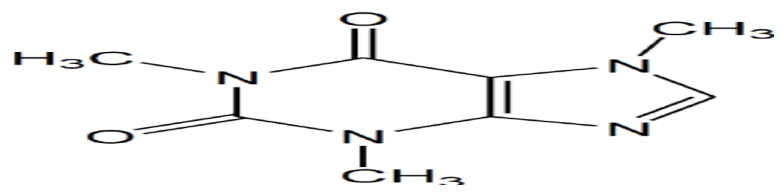

Fig6. Caffeine 


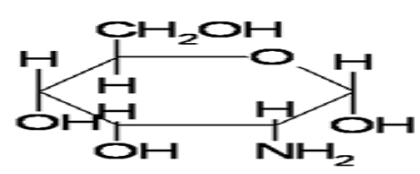

Fig7. Glucosamine

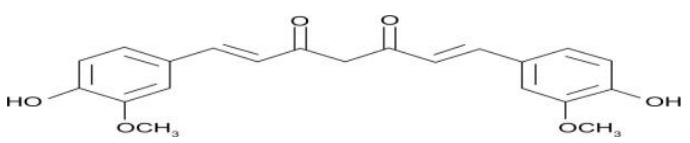

Fig9. Curcumin

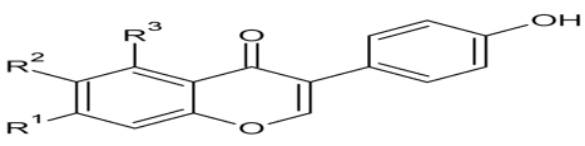

Fig11. Isoflavone<smiles>NC(CCC(=O)NC(CS)C(=O)NCC(=O)O)C(=O)O</smiles>

Fig8. Glutathione

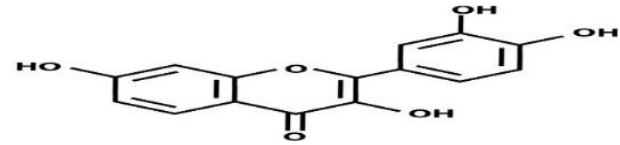

Fig10. Flavonoids

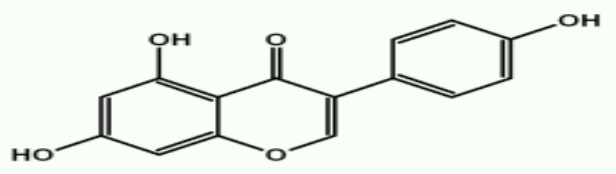

Fig12. Genistein

\section{CONCLuSion}

This present review has diagonised recent advances in nutraceuticals and the crucial role of nutraceuticals in prevention and treatment of various diseases. Most people prefer the treatment of any ailment in a natural way which can be achieved by including nutraceuticals in regular use. The consumption of nutraceuticals provides best option to stay natural and to improve the quality of life. In addition, nutraceuticals can also provide an alternative for patients who are unwilling to go through chemical therapy. It is also advantageous over other therapies in terms of cost. The studies reveal the fact that nutraceuticals exert different types of biological activities in mechanized manner. These properties ultimately lower the age related and chronic diseases. Presently, large numbers of such potential nutraceuticals are undergoing the phases of research and development. The marketing graph of nutraceuticals is also rising all over the world. The market values reported depicts the public behavior of giving more emphasis on the use of nutraceuticals which ultimately is related to the brighter scope of nutraceutical industry. However, there is a huge scope for further advancement in the field by introducing delivery carrier and further spreading awareness about the potential of such systems. Therefore, the field undoubtedly has the greatest hidden value associated with it, which is just needed to be explored to provide easy and better healthy life.

Nutraceuticals are destined to play an important role in future therapeutic developments but their success will be governed by control of purity, safety and efficacy without inhibiting innovation. Nutraceuticals will continue to appeal because they are convenient for today's lifestyle. Some are also genuinely researched and offer novel ingredients that can bring about health benefits quicker than would normally be the case through eating conventionally healthy foods alone. The present accumulated knowledge about nutraceuticals represents undoubtedly a great challenge for nutritionists, physicians, food technologists and food chemists.

\section{AUTHOR CONTRIBUTIONS}

Iyen Ifeoma S., conceived the idea, reviewed all the articles and wrote the manuscript. Godwin Ogofotha, Oghenekeno and Oko, John Odiba afforded some preliminary information on nutraceuticals for review.

\section{REFERENCES}

[1] Kalra EK. (2003) Nutraceutical - Definition and introduction. American Association of Pharmaceutimcal Scientists; 5:25.

[2] Zhao J. Bentham Science Publishers; 2007. [Last accessed on 2018 Dec 24]. Nutraceuticals, Nutritional Therapy, Phytonutrients, and Phytotherapy for Improvement of Human Health: A Perspective on Plant Biotechnology Application. Available from

[3] Chauhan B, Kumar G, Kalam N, Ansari SH. (2013 Current concepts and prospects of herbal nutraceutical: A review. Journal of Advanced Pharmaceutical Technology \& Research; 4:4-8. 
[4] Zeisel SH.(1999) Regulation of "nutraceuticals" Science;285:1853-5.

[5] Hardy G.(2000) Nutraceuticals and functional foods: Introduction and meaning. Nutrition; 16:3

[6] Khosravi-Boroujeni H, Mohammadifard N, Sarrafzadegan N, Sajjadi F, Maghroun M, Khosravi A, et al.(2012) Potato consumption and cardiovascular disease risk factors among Iranian population. International Journal of Food Sciences and Nutrition.;63:913-20.

[7] Shirzad H, Burton RC, Smart YC, Rafieian-kopaei M, Shirzad M.(2011) Natural cytotoxicity of NC$2+$ cells against the growth and metastasis of WEHI-164 fibrosarcoma. Scand. Journal of Immunology.;73:85-90.

[8] Baradaran A.(2012) Beyond mineral metabolism, the bright immunomodulatory effect of vitamin D in renal disease. Journal of Nephropharmacology.;1:17-8.

[9] Nasri H, Behradmanesh S, Ahmadi A, Rafieian-Kopaei M.(2014) Impact of oral vitamin D (cholecalciferol) replacement therapy on blood pressure in type 2 diabetes patients; a randomized, doubleblind, placebo controlled clinical trial. Journal of Nephropathology.;3:29-33.

[10] Heidarian E, Rafieian-Kopaei M.(2013) Protective effect of artichoke (Cynara scolymus) leaf extract against lead toxicity in rat. Pharmaceutical Biology.;51:1104-9.

[11] Nasri H, Rafieian-Kopaei M.(2014) Protective effects of herbal antioxidants on diabetic kidney disease. Journal of Research in Medical Sciences.;19:82-3.

[12] Willis MS, Wians FH.(2003) The role of nutrition in preventing prostate cancer: A review of the proposed mechanism of action of various dietary substances. International Journal of Clinical Chemistry.;330:5783.

[13] Shirzad H, Kiani M, Shirzad M.(2013) Impacts of tomato extract on the mice fibrosarcoma cells. Journal of HerbMed Pharmacology2:13-6.

[14] Stahl W, Sies H.(2005) Bioactivity and protective effects of natural carotenoids. Biochimica et Biophysica Acta (BBA); 1740:101-7.

[15] 15.Shirzad H, Taji F, Rafieian-Kopaei M.(2011) Correlation between antioxidant activity of garlic extracts and WEHI-164 fibrosarcoma tumor growth in BALB/c mice. Journal of Medicinal Food.;14:969-74.

[16] Shirzad H, Shahrani M, Rafieian-Kopaei M.(2009) Comparison of morphine and tramadol effects on phagocytic activity of mice peritoneal phagocytes in vivo. International Immunopharmacology.;9:968-70

[17] Limer JL, Speirs V.(2004) Phyto-oestrogens and breast cancer chemoprevention. Breast Cancer Research.;6:119-27.

[18] Thomasset SC, Berry DP, Garcea G, Marczylo T, Steward WP, Gescher AJ.(2007) Dietary polyphenolic phytochemicals - Promising cancer chemopreventive agents in humans? A review of their clinical properties. International Journal of Cancer.;120:451-8.

[19] Li H, Wang Z, Liu Y.(2003) Review in the studies on tannins activity of cancer prevention and anticancer. Journal of Chinese medicinal materials. 26:444-8.

[20] Nasri H, Sahinfard N, Rafieian M, Rafieian S, Shirzad M, Rafieian-kopaei M.(2014) Turmeric: A spice with multifunctional medicinal properties. Journal of HerbMed Pharmacology.;3:5-8.

[21] Higdon JV, Delage B, Williams DE, Dashwood RH.(2007) Cruciferous vegetables and human cancer risk: Epidemiologic evidence and mechanistic basis. Pharmacological Research.;55:224-36.

[22] Tamadon MR, Baradaran A, Rafieian-Kopaei M.(2014) Antioxidant and kidney protection; differential impacts of single and whole natural antioxidants. Journal of renal injury prevention..;3:41-2

[23] Tavafi M.(2013) Diabetic nephropathy and antioxidants. Journal of nephropathology:;2:20-7.

[24] Rafieian-Kopaei M, Nasri H.(2013) Ginger and diabetic nephropathy. Journal of renal injury prevention.;2:9-10.

[25] Brouns F.(2002) Soya isoflavones: A new and promising ingredient for the health foods sector. Food Research International.;35:187-93

[26] Baradaran A.(2012) Lipoprotein (a), type 2 diabetes and nephropathy; the mystery continues. Journal of nephropathology.;1:126-9.

[27] Tolouian R, T Hernandez G.(2013) Prediction of diabetic nephropathy: The need for a sweet biomarker. Journal of nephropathology.;2:4-5.

[28] Sirtori CR, Galli C.(2002) N-3 fatty acids and diabetes. Biomedicine \& Pharmacotherapy;56:397-406.

[29] 29.Momeni A(2012)Serum uric acid and diabetic nephropathy.Journal of renal injury prevention;1:37-8.

[30] 30. Brookmeyer R, Johnson E, Ziegler-Graham K, Arrighi HM.(2007) Forecasting the global burden of Alzheimer's disease. Alzheimer's \& Dementia.;3:186-91.

[31] 31. Gupta P, Andrew H, Kirschner BS, Guandalini S.(2000). Is lactobacillus GG helpful in children with Crohn's disease? Results of a preliminary, open-label study. Journal of Pediatric Gastroenterology and Nutrition.;31:453-7.

[32] 32. Fuller R.(1991) Probiotics in human medicine. Gut.;32:439-42. 
[33] 33.Hajivandi A(2014) Amiri M.World kidney day 2014:Kidney disease and elderly. Journal of Parathyroid Disease.;2:3-4

[34] 34. Shahbazian H.(2013) World diabetes day; 2013. Journal of renal injury prevention.;2:123-4.

[35] 35. Gharipour M, Ramezani MA, Sadeghi M, Khosravi A, Masjedi M, Khosravi-Boroujeni H, et al. Sex based levels of C-reactive protein and white blood cell count in subjects with metabolic syndrome: Isfahan Healthy Heart Program. Journal of Research in Medical Sciences. 2013;18:467-72.

[36] 36. Nasri H, Motamedi P, Dehghani N, Nasri P, Taheri Z, Kinani F, et al.(2014) Vitamin D and immune system. Journal of Renal Endocrinology.;1:5-7.

[37] 37. Asgary S, Kelishadi R, Rafieian-Kopaei M, Najafi S, Najafi M, Sahebkar A.(2013) Investigation of the lipid-modifying and antiinflammatory effects of Cornus mas L. supplementation on dyslipidemic children and adolescents. Pediatric Cardiology:;34:1729-35.

[38] 38. Iriti M, Faoro F.(2006) Grape phytochemicals: A bouquet of old and new nutraceuticals for human health. Medical Hypotheses.;67:833-8.

[39] 39. Garg A, Garg S, Zaneveld LJ, Singla AK.(2001) Chemistry and pharmacology of the Citrus bioflavonoid hesperidin. Phytotherapy Research.;15:655-69.

[40] 40. Rafieian-Kopaei M, Baradaran A, Rafieian M.(2013) Plants antioxidants: From laboratory to clinic. Journal of nephropathology:;2:152-3.

[41] 41.Ghayur MN,Gilani AH,Afridi MB,Houghton PJ(2015)Cardiovascular effects of ginger aqueous extract and its phenolic constituents are mediated through multiple pathways. Vascular Pharmacology;43:234-41.

[42] 42. Bahmani M, Vakili-Saatloo N, Gholami-Ahangaran M, Karamati SA, Khalil-Banihabib E, Hajigholizadeh GH, et al.(2013) A comparison study on the anti-leech effects of onion (Allium cepa L) and ginger (Zingiber officinale) with levamisole and triclabendazole. Journal of HerbMed Pharmacology.;2:13.

[43] 43. Nasri H, Nematbakhsh M, Ghobadi SH, Ansari R, Shahinfard N, Rafieian-kopaei M.(2013) Preventive and curative effects of ginger extract against histopathologic changes of gentamicin-Induced tubular toxicity in rats. International Journal of Preventive Medicine.;4:316-21.

[44] 44. Gita C.(2004) Functional food attributes of n-3 polyunsaturated and conjugated linoleic acid enriched chicken eggs. Current Topics in Nutraceutical Research.;2:113-21.

[45] 45. Khosravi-Boroujeni H, Mohammadifard N, Sarrafzadegan N, Sajjadi F, Maghroun M, Khosravi A, et al.(2012) Potato consumption and cardiovascular disease risk factors among Iranian population. International Journal of Food Sciences and Nutrition.;63:913-20.

[46] 46. Sidhu KS.(2003) Health benefits and potential risks related to consumption of fish or fish oil. Regulatory Toxicology and Pharmacology.;38:336-44.]

[47] 47. Heidarian E, Rafieian-Kopaei M, Ashrafi K.(2013) The Effect of hydroalcoholic extract of Allium latifolium on the liver phosphatidate phosphatase and serum lipid profile in hyperlipidemic rats. Journal of Babol University of Medical Sciences; 15:37-46.

[48] 48. Kruger CL, Murphy M, DeFreitas Z, Pfannkuch F, Heimbach J.(2002) An innovative approach to the determination of safety for a dietary ingredient derived from a new source: Case study using a crystalline lutein product. Food and Chemical Toxicology (FCT); 40:1535-49.

[49] 49. Rabiei Z, Rafieian-Kopaei M, Heidarian E, Saghaei E, Mokhtari S. (2014) Effects of Zizyphus jujube extract on memory and learning impairment induced by bilateral electric lesions of the nucleus basalis of Meynert in rat. Neurochemical Research.;39:353-60.

[50] 50. Losso JN. (2003) Targeting excessive angiogenesis with functional foods and nutraceuticals. Trends in Food Science \& Technology; 14:455-68.

[51] 51. Anwar F, Latif S, Ashraf M, Gilani AH. (2007) Moringa oleifera: A food plant with multiple medicinal uses. Phytotherapy Research.21:17-25.

[52] 52. Caterson ID, Gill TP. (2002) Obesity: Epidemiology and possible prevention. Best Practice \& Research Clinical Endocrinology \& Metabolism.;16:595-610.

[53] 53. Rubin SA, Levin ER. (1994) Clinical review 53: The endocrinology of vasoactive peptides: Synthesis to function. Journal of Clinical Endocrinology and Metabolism.;78:6-10.

[54] 54. Boozer CN, Nasser JA, Heymsfield SB, Wang V, Chen G, Solomon JL. (2001) An herbal supplement containing Ma Huang-Guarana for weight loss: A randomized, double-blind trial. International journal of obesity and related metabolic disorders.;25:316-24.

[55] 55. Rafieian-Kopaei M. (2014) Identification of medicinal plants affecting on headaches and migraines in Lorestan Province, West of Iran. Asian Pacific Journal of Tropical Medicine.;7:376-9.

[56] 56. Jang M, Cai L, Udeani GO, Slowing KV, Thomas CF, Beecher CW, et al. (1997) Cancer chemopreventive activity of resveratrol, a natural product derived from grapes. Science.;275:218-20. 
[57] 57. Rouhi-Broujeni A, Heidarian E, Darvishzadeh-Boroojeni P, Rafieian-Kopaei M, Gharipour M. (2013) Lipid lowering activity of moringa pergerina seeds in rat: A comparison between the extract and atorvastatin. Research Journal of Biological Sciences.;8:150-4.

[58] 58. Nasri H, Ardalan MR, Rafieian-Kopaei R. (2014) On the occasion of world hypertension day 2014. Journal of Parathyroid Disease.;2:19-20.

[59] 59. Hathcock J. (2001) Dietary supplements: How they are used and regulated. Journal of Nutrition; 131:1114-7.

[60] 60. Mobarhan S. (1994) Micronutrient supplementation trials and the reduction of cancer and cerebrovascular incidences and mortality. Nutrition Reviews; 52:102-5.

[61] 61. Agus DB, Gambhir SS, Pardridge WM, Spielholz C, Baselga J, Vera JC, et al. (1997) Vitamin C crosses the blood brain barrier in the oxidized form through the glucose transporters. Journal of Clinical Investigation.;100:2842-8.

[62] 62. Jialal I, Fuller CJ. (1995) Effect of vitamin E, vitamin C and beta carotene on the LDL oxidation and atherosclerosis. Canadian Journal of Cardiology (CJC).;11:97-103.

[63] 63. Klein C, Sato T, Meguid MM, Miyata G. (2000) From food to nutritional support to specific nutraceuticals: a journey across time in the treatment of disease. Journal of Gastroenterology; 35:1-6.

[64] 64. Mathew P, Wyllie R, Van Lente F, Steffen RM, Kay MH. (1996) Antioxidants in hereditary pancreatitis. American Journal of Gastroenterology.;91:1558-62.

[65] 65. Clark LC, Combs Jr GF, Turnbull BW, Slate EH, Chalker DK, Chow J, et al. (1996). Effects of selenium supplementation for cancer prevention in patients with carcinoma of the skin. A randomized controlled trial. Nutritional Prevention of Cancer Study Group. Journal of the American Medical Association.;276:1957-63.

[66] 66. Ceremuzynski L, Chamiec T, Herbaczynska-Cedro K. (1997) Effect of supplemental oral L-arginine on exercise capacity in patients with stable angina pectoris. American Journal of Cardiology.;80:331-3.

[67] 67. Braeckman J.(1994) The Extract of Serenoa repens in the treatment of benign prostatic hyperplasia: a multicenter open study. Current Therapeutic Research (CTR).;55:776-85.

[68] 68. Melchart D, Linde K, Worku F, Sarkady L, Holzmann M, Jurcic K, et al. (1995) Results of five randomized studies on the immunomodulatory activity of preparation of Echinacea. Journal of Alternative and Complementary Medicine; 1:145-60.

[69] 68. Grammatikos AP (2008). The genetic and environmental basis of atopic diseases. Annals of Medicine.;40:482-95

Citation: Ifeoma Sandra., et al., (2019). Advances in Nutraceuticals. International Journal of Medicinal Plants and Natural Products (IJMPNP), 5(2), pp.8-22. http://dx.doi.org/10.20431/2454-7999.0502002

Copyright: (C) 2019 Authors, this is an open-access article distributed under the terms of the Creative Commons Attribution License, which permits unrestricted use, distribution, and reproduction in any medium, provided the original author and source are credited. 